\title{
EVALUACIÓN EN EL PROCESO EDUCATIVO
}

Abog. Liliana Seminario Méndez

\begin{abstract}
RESUMEN
EI presente artículo tiene por finalidad ver la importancia de la evaluación en el proceso educativo e indicar algunas herramientas de evaluación aplicables en Derecho para lograr que nuestras estudiantes exterioricen su personalidad para fortalecer sus capacidades $y$ hacer de ellas profesionales exitosas.
\end{abstract}

\section{ABSTRACT}

This research aims to highlight the importance of the educational process evaluation and to explain sorne evaluation tools applicable to the career of Law in order that our students externalize their personality strengthen their abilities and become successful professional lawyers.

\section{PALABRAS CLAVE}

Evaluación, instrumento, conocimiento, aprendizaje, competencias.

\section{KEYWORDS}

Evaluation, tool, knowledge, learning, competences.

\section{INTRODUCCIÓN}

A nte todo, debo empezar señalando que el integrar parte de una universidad y el ser docente ele una entidad educativa superior resulta de mucha satisfacción cuando tomamos conciencia que en nuestras manos está el formar profesionales de calidad con sólidos valores y conocimientos jurídicos y éticos. Para ello, contamos con una estructura curricular adaptada a la realidad, con docentes de amplia experiencia en las materias que enseñan y con conocimientos de didáctica universitaria, con estudiantes dispuestas a ser profesionales eleéxito, entre otras variables.

La evaluación es parte importante y sensible de todo proceso de enseñanza - aprendizaje y para aplicarla tenemos muchos instrumentos que nos llevarán a conocer a cada una de nuestras estudiantes y resaltar en ellas sus capacidades, habilidades, competencias, con la finalidad de obtener como resultado final una profesional exitosa y competente en el- mercado laboral. 


\section{tCómo debe ser la evaluación?}

La evaluación debe ser objetiva y transparente, teniendo como meta medir los conocimientos de las estudiantes; debe ser formativa y construida de tal forma que pueda llegar a cambiarse para mejorar, ella es un canúno de comunicación entre las estudiantes y los docentes que sirve para identificar debilidades $\mathrm{y}$ fortalezas.

Adicionalmente, la evaluación debe darse oportunamente y con la certeza que lo que se pregunta tiene un sentido. Son muchas cosas las que sedeben que tener presentes al elaborar los objetivos de la evaluación de un curso, de ellos depende el desarrollo de toda una materia que ampliará los conocimientos de nuestras estudiantes.

\section{tQué debemos tener en cuenta para aplicar herramientas de evaluación?}

Todo docente, de manera responsable, establece en el sílabo que regirá durante el semestre, las herranúentas de evaluación que aplicar( de ahí se desprende que de acuerdo al propósito del maestro se plasmará la forma cómo evaluará a sus estudiantes.

Pero previamente el docente para planificar su sistemadeevaluación tendrá que hacer una selección de técnicas y procedimientos para llegar a aprehender los conocimientos, capacidades y aptitudes ele los estudiantes; tendrá que marcar unidades o temas a evaluar y esperar oportunamente elmomento en el cual aplicar la evaluación.

Posteriormente, una vez aplicada la evaluación, de acuerdo al instrumento escogido, podemos saber si nuestras estudiantes avanzan en el curso y si estamos sentando conocimientos para continuar; en caso contrario, considero, debe replantearse el tema evaluado mejorando el proceso de aprendizaje o las técnicas con las cuales se evaluó.

lCuándo se puede evaluar?

La evaluación puede darse en cualquier momento; si la aplicamos al inicio de ciclo nos demuestra como está la alumna y si sus conocimientos previos son sólidos o necesitan un refuerzo para continuar avanzando, sobretodo si se trata ele cursos secuenciales.

Eldocentepuedeevaluarunidades, con ello analiza el avance en el proceso enseñanza - aprendizaje, con la finalidad de continuar, mejorar o replantear, de esa forma detectará las deficiencias o los logros alcanzados y estará a tiempo de subsanar cualquier vacío o error en la captación del conocimiento.

En una evaluación aplicada al final del curso, generalmente, se ve el horizonte llegándose a una plena, mediana o escasa satisfacción frente a los resultados.

Es por ello que la evaluación dentro del currículo es una de las partes más delicadas que todo docente debe tener presente y saber, de acuerdo al curso que dicta, cómo incidir para tener los mejores resultados de sus estudiantes, entendiéndose lograr la mayor captación de conocimiento que se brinda, ya que al tener los resultados como indicador del avance ele un curso y del entendimiento del mismo ele parte ele los estudiantes, hará que continuemos o replanteemos. La rigurosidad que se pueda tener al momento de una evaluación dependerá del docente, no siendo fácil en algunos casos el evaluar.

\section{La importancia de evaluar por competencias}

La evaluación es indispensable porque forma parte de ese proceso educativo que durante el dictado de un curso nos da a conocer el impacto de nuestras enseñanzas, pudiendo evaluarse con un sinfín de herramientas de las que posteriormente mencionaré algunas aplicables al Derecho.

Hoy en día todos debemos estar inmersos en llevar a cabo una enseñanza y, por ende, una evaluación ele competencias, la que tiene como objetivo determinar qué tanto se domina una capacidad, la misma que se basa en indicadores pre-constituidos y aceptados, llegando a establecerse los logros alcanzados y aquellos aspectos en que sin estar mal, debemos mejorar, buscando siempre que la estudiante se trace como reto el ir avanzando y optimizar continuamente susconocimientos.

La evaluación por competencias depende mucho de los estudiantes toda vez que en su actuar debe autorregularse en torno al aprendizaje que va adquiriendo, demostrando un alto nivel de autonomía, compromiso y responsabilidad.

Pero para llegar al reconocimiento social de las competencias de un estudiante debemos formarlo con sentido crítico, participativo, democrático e ir desarrollando sus habilidades, destrezas, aptitudes, afectos y valores. 


\section{Algunas herramientas de evaluación aplicables al Derecho}

Mencionaré algunas herramientas de evaluación utilizadas en Derecho y que tienen por finalidad la formación integral de nuestras estudiantes:

Intervenciones en clases, los docentes, de acuerdo a las preguntas expuestas, sabrán cuanto van adelantando en el curso y si es que sus enseli.anzas están siendo captadas correctamente por las estudiantes; tendrá conocimiento de la profundidad en el aprendizaje pudiendo llegar a calificar inclusive la expresión oral de la alumna, a su vez ella demostrará su interés y dominio en elcurso.

Las exposiciones que nos permiten pulir su estilo,su oratoria, lo que para una estudiante de Derecho es de gran importancia, ya que ellas cuando salgan ele las aulas universitarias se dedicarán a distintas actividades en donde si.empre tendrán que expresarse verbalmente y deben hacerlo de la forma más clara posible, adecuada y coherente. El hablar en público sobre un determinado terna y poder refutar su planteamiento enriquece su personalidad $\mathrm{y}$ fortalece su espíritu haciéndolas cada vez más seguras de sí; para ello se preparan a profundidad y llegan a sentir la satisfacción que da el conocimiento.

Los exámenes escritos, los mismos que son considerados clásicos, pero son los instrumentos que nos clan a conocer el estilo ele redacción de las estudiantes y es ahí donde nos damos cuenta de errores ortográficos, ele redacción gramatical que pudieran tener y que apuntamos a mejorar mediante cursos. Generalmente, los exámenes escritos son el reflejo de las enseli.anzas del docente y éste incide en ternas concretos, de ahí se desprende el nivel ele captación de la estudiant e; en cambio, si tomamos controles ele lectura, que es otro instrumento empleado para reforzar algunos temas, nos darnos cuenta ele que tan buena memoria tienen nuestras estudiantes y ele su nivel ele comprensión lectora.

Estos exámenes escritos tienen diversas variantes entre las que encontramos los exámenes ele conocimientos en donde las estudiantes contestan teóricamente a las interrogantes formuladas por el docente en base a un tema extenso y se tiende a dar cierto plazo para su desarrollo. Los exámenes prácticos en los que se plantean diversas situaciones a resolver y que en Derecho apun tan a la resolución de casos. Los exámenes mixtos, que es la mezcla ele ambos exámenes, es decir, hay una combinación elela parte teórica con la solución ele problemas en base a las clases expuestas y se ve la capacidad resolutiva de la estudiante. Los exámenes objetivos, que desde mi punto ele vista son memorísticos ya que hay un enunciado y para el una sola respuesta, este tipo de exámenes no da libertad de exp resión a las estudiantes.

Los seminarios que permiten que las estudiantes interactúen, formándo se debates alrededor del tema a analizar, eso ayuda a que dem uestr en la profundidad de sus conocimientos y su capacidad de expresión se fortalece; con ello se fomenta la argumentación yla rapidez mental.

La solución de casos reales y ele problemas jurídicos, de suma importancia para quienes desean ser abogadas, pues permite desarrollar su capacidad y habilidad de pensamiento, las estudian tes puede $n$ aplicar todos sus conocimientos a fin ele lograr absolver jurídicamente un caso. Ellas conjugarán todas las máterias para llegar a la solución del problema, legalmente hablando, lograrán identificar el problema, a que materia correspon de, la norma vigente que se debe aplicar para una mejor solución del caso concreto. Resulta interesante porque se evalúa su capacidad ele análisis y el saber hilar entre las variadas materias del Derecho.

El análisis de jurisprudencia, en donde las estudiantes interpretan y analizan a la luz de la normatividad y la doctrina las resoluciones judiciales a partir de la revisión ele casos controvertidos. Identifican distintas figuras procesales y sustantivas, así como sus contenidos comparando diferentes sentencias.

Los talleres en los que se demuestra la capacida d de trabajo individual y grupal, asimismo se demuestra la comprensión sobre temas determinados expuestos con anterior idad.

El debate que es una prueba de comunicación oral que permite a las estudiantes ar gu me nt ar jur!dicamente sobre un tema o caso y dar la solución al mismo, las estudiantes demostrarán su dominio del terna y la preparación antelacla a la presentación; ele la misma forma demostrarán su rapidez mental para poder refutar los argumentos contrarios.

Los trabajos monográficos nos mostrarán la capacidad para investigar ele nuestras estudian tes, debiendo tener cuidado en la redacción y en el contenido. Para llegar a un buen traba jo monográfico las estudiantes tendrán que haber 
recurrido a mucha lectura para darle el enfoque correspondiente; en algunos temas es importante acudir al muestreo para poder verificar nuestro planteamiento .

lCómo tomar los resultados de una evaluación?

De los resultados de la evaluación los docentes podemos estar satisfechos con nuestro desempeño como tales, medianamente satisfechos o insatisfechos, porque son el reflejo del proceso de enseñanza-aprendizaje que tenemos en un lapso y sobre uncurso determinado .

Frente a los resultados de una evaluación nos sentiremos satisfechos porque las notas son aprobatorias, o no, porque son insuficientes, pero siempre debemos tener presente que el preparar un curso y el evaluarlo no es juego, debemos preguntarnos porque incluimos una urúdad y cómo la evaluaremos, hacia donde nos dirigimos con tal o cual pregunta, es oportuno evaluar ahora luego de algunas semanas, creará ventajas para algunas estudiantes elevaluar sobre determinados temas.

Si queremos tener la satisfacción de la labor bien cumplida, preocupémonos de que las herramientas aplicables para evaluar sean las adecuadas para logr ar una formación integral de nuestras estudiantes, démosles la oportunidad de crecer con cada curso y de desarrollar sus habilidades; así lograremos hacer de ellas las profesionales exitosas que la UNIFÉ está acostumbrada a tener.

\section{REFERENCIAS}

Autores varios. Evaluación educativa. Evaluación del aprendizajeyelelasinstitucioneseducativas.

SosaEspinoza, Myriam Janct. Laevaluación del aprendizajey dela enscrianza: procedimientose instrumentos.

Wong F., Margarita. Estrategias didácticas: estrategias de enseñanza y de aprend izaje.

\footnotetext{
Abog. Liliana Seminario Méndez

Abogada egresada de la Facultad de Derecho de la UNIFÉ, con estudios de Maestría en Derecho Civil con mención en Derecho de Familia. Es Directora del Programa Académico de Derecho de la UNIFÉ y socia del Estudio Beltrán \& SeminarioAbogadosAsociados .
} 